\title{
DETECÇÃO DE BAIXOS NÍVEIS CARBURIZAÇÃO EM TUBOS DE REFORMA USANDO UM SENSOR MAGNÉTICO*
}

\author{
Mónica P. Arenas ${ }^{1}$ \\ Juan Esteban Correa Lopez ${ }^{2}$ \\ Clara Johana Pacheco ${ }^{3}$ \\ Luiz Henrique de Almeida ${ }^{4}$ \\ Carlos Bruno Eckstein ${ }^{5}$ \\ Laudemiro Nogueira Junior ${ }^{6}$ \\ João Marcos A. Rebello \\ Gabriela Ribeiro Pereira ${ }^{8}$
}

\section{Resumo}

Aços austeníticos resistentes ao calor apresentam propriedades mecânicas que permitem seu uso em altas temperaturas e pressão, sendo frequentemente utilizados em fornos de reforma e pirolise. Devido às severas condições de operação danos de carburização podem ser induzidos. No presente trabalho foram analisados corpos de prova provenientes de fornos de reforma que apresentaram baixos níveis carburização. Com a finalidade de desenvolver uma metodologia que permita detectar e separar diferentes níveis de carburização, foi projetado, simulado e construído um sensor magnético constituído por um yoke e um sensor Hall. De acordo aos resultados obtidos, concluiu-se que o sensor permitiu determinar confiavelmente 0 nível de carburização em ligas austeníticas que estiveram expostas a diferentes temperaturas de operação.

Palavras-chave: Carburização; Fornos de reforma; Simulação FEM; Sensor magnético.

\section{DETECTION OF LOW CARBURIZATION LEVEL IN REFORMER TUBES USING A MAGNETIC SENSOR}

\section{Abstract}

Heat-resistant austenitic stainless steels has mechanical properties that allow their use at high temperatures and pressures, being frequently used in reformer and pyrolysis furnaces. Due to the harsh operational conditions, carburization damages may be induced. This study aims to study samples coming from a reformer tube that presented low carburization levels. In order to develop a methodology to detect and separate different carburization levels, a magnetic sensor, composed of a yoke and a Hall sensor, was designed, simulated and built. According to the results obtained, it was concluded that the sensor allowed to determine the carburization level in austenitic alloys that were exposed to different operational temperatures.

Keywords: Carburization; Reformer furnaces; FEM simulation; Magnetic sensor.

1 MSc. em Engenharia Metalúrgica e de Materiais, estudante de doutorado, Universidade Federal do Rio de Janeiro, Rio de Janeiro, Brasil.

2 Engenheiro Físico, estudante de mestrado, Universidade Federal do Rio de Janeiro, Rio de Janeiro, Brasil.

3 DSc. em Física, pesquisadora, Laboratório de Ensaios Não Destrutivos Corrosão e Soldagem, Rio de Janeiro, Brasil.

4 DSc. em Engenharia Metalúrgica e de Materiais, professor do programa Engenharia Metalúrgica e de Materiais, Universidade Federal do Rio de Janeiro, Rio de Janeiro, Brasil.

5 DSc. em Engenharia Metalúrgica e de Materiais, PETROBRAS, Rio de Janeiro, Brasil.

6 MSc. em Engenharia Civil, PETROBRAS, Rio de Janeiro, Brasil.

7 DSc. em Metalurgia Física, professor do programa Engenharia Metalúrgica e de Materiais, Universidade Federal do Rio de Janeiro, Rio de Janeiro, Brasil.

8 DSc. em Engenharia Nuclear, professor do programa Engenharia Metalúrgica e de Materiais, Universidade Federal do Rio de Janeiro, Rio de Janeiro, Brasil. 


\section{INTRODUÇÃO}

Tubos de reforma a vapor são expostos a altas temperaturas e pressão durante o tempo em serviço, são comumente feitos de ligas austeníticas a partir de materiais fundidos por centrifugação com altos teores de cromo e níquel, tipicamente da família HK e HP $(1,2)$. A fundição centrífuga produz uma estrutura mais uniforme e os grãos são orientados segundo a direção radial proporcionando uma maior resistência à fluência (2).

Paradas não planejadas do forno e/ou perdas de produção causadas por falhas nos tubos podem ser extremamente custosas e devem ser minimizadas sempre que possível. Desta forma, a previsão da condição estrutural e da vida útil das colunas reformadoras torna-se de vital importância. A inspeção e avaliação destas estruturas é normalmente realizada durante as paradas programadas para manutenção através de técnicas não destrutivas como por exemplo perfilometría a laser (3), correntes parasitas (4) e/ou ultrassom (5). As principais causas de ocorrência de falha dos tubos de reforma são fluência, superaquecimento, surto de temperatura, deposição de coque, carburização, oxidação, entre outros. No entanto, o presente trabalho foca-se no desenvolvimento de uma metodologia para identificar danos de carburização.

O fenômeno de carburização acarreta a redução da ductilidade da liga, o aumento da tensão interna na camada carburizada afetando negativamente a resistência à fluência e aos ciclos térmicos $(6,7)$. Dependendo do nível de carburização, a vida útil das colunas pode diminuir ou ainda apresentar falhas prematuras. Mesmo que os danos de carburização ocorram principalmente nos tubos de pirolise, os tubos de reforma também apresentam deposição e difusão de carbono na parede interna. Sendo importante desenvolver uma metodologia que permita caracterizar e medir a presença deste dano. Quando estas ligas apresentam danos de carburização, a matriz austenítica sofre um empobrecimento de cromo e consequentemente começa a exibir um comportamento ferromagnético (7-9), característica que indica a viabilidade do uso de sensores magnéticos para a detecção da carburização.

Os corpos de prova do presente estudo foram retirados de uma coluna reformadora a qual apresentou sinais com baixos níveis de carburização $(<800 \mu \mathrm{m})$. Para determinar a presença de carburização foi desenvolvida uma metodologia baseada na projeção, simulação e construção de um sensor magnético. A simulação computacional, desenvolvida no COMSOL Multiphysics, possibilitou otimizar a geometria e as propriedades magnéticas do sensor. O sensor magnético permitiu estabelecer uma relação entre a densidade do campo magnético e a presença da camada carburizada.

\section{MATERIAIS E MÉTODOS}

\subsection{Corpos de prova}

O presente trabalho usou como material de estudo uma coluna reformadora após aproximadamente 130.000 horas em serviço. Foram retiradas três amostras de diferentes seções da coluna, expostas a diferentes temperaturas de operação, cada 
uma com diferente grau de carburização e envelhecimento, conforme mostrado na Tabela 1.

Tabela 1. Características das amostras analisadas

\begin{tabular}{|c|c|c|}
\hline Amostra & $\begin{array}{c}\text { Estado de } \\
\text { envelhecimento }\end{array}$ & $\begin{array}{l}\text { Dimensão do } \\
\text { tubo }(\mathrm{mm})\end{array}$ \\
\hline A & I & \multirow{3}{*}{$\begin{array}{c}\phi \times e \times h \\
133 \times 15 \times 200\end{array}$} \\
\hline B & III & \\
\hline C & V & \\
\hline
\end{tabular}

A composição química da liga foi determinada com as técnicas de fluorescência de raios-X e de espectroscopia de plasma. Conforme observado na Tabela 2, todos os elementos da liga estão conforme a norma ASTM A-297 (10), no entanto, o Nb e o Ti são elementos adicionados indicando que o material analisado é referente a uma liga HP modificada (HP-NbTi).

Tabela 2. Composição química da liga

\begin{tabular}{lcccccccccc}
\hline & $\mathbf{C r}$ & $\mathbf{N i}$ & $\mathbf{C}$ & $\mathbf{M n}$ & $\mathbf{S i}$ & $\mathbf{P}$ & $\mathbf{S}$ & Mo & $\mathbf{N b}$ & $\mathbf{T i}$ \\
\hline peso\% & 26,1 & 34,3 & 0,52 & 1,26 & 1,89 & 0,023 & 0,012 & 0,018 & 1,26 & 0,010 \\
\hline$\% \mathrm{Fe} \mathrm{Bal}$ & & & & & & & & & &
\end{tabular}

\subsection{Caracterização microestrutural}

A caracterização microestrutural da camada carburizada foi realizada com o microscópio eletrônico de varredura Jeol JSM 6460LV no modo retroespalhado (BSE). As amostras foram retiradas da seção transversal de cada tubo e preparadas metalograficamente.

\subsection{Simulação por elementos finitos - COMSOL Multyphysics}

O modelo computacional foi desenvolvido no software por elementos finitos COMSOL Multiphysics $\AA$, utilizando o modelo magnetostático para um espaço tridimensional. A simulação permitiu otimizar a geometria e os parâmetros mais adequados para a construção do sensor.

\section{RESULTADOS E DISCUSSÃO}

\subsection{Caracterização Metalográfica}

A carburização promove a formação massiva de carbetos de cromo na região próxima à parede interna mudando a microestrutura do aço, criando novos carbetos e favorecendo a coalescência dos já existentes (6). Dita precipitação leva ao empobrecimento de cromo na matriz tornando-a ferromagnética $(7,8)$. Com o objetivo de medir a espessura da camada carburizada, foi construído um mosaico com as imagens adquiridas no MEV no modo de elétrons retroespalhados (BSE). $\mathrm{Na}$ Figura 1 observa-se uma região mais escura composta por diferentes tipos de óxidos e coque (4), seguida pela camada carburizada que apresenta 0 coalescimento e crescimento dos carbetos de cromo. 


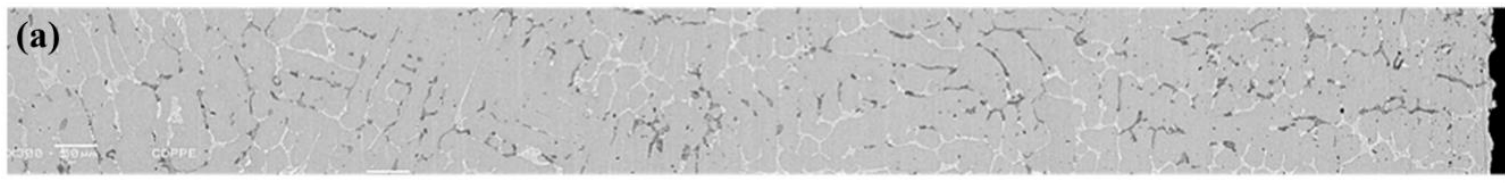

(b)

(c)

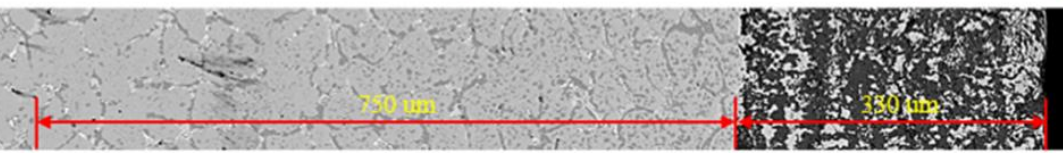

Figura 1. Metalografias MEV no modo retroespalhado próxima à parede interna nas amostras com estado de envelhecimento (a) I, (b) III e (c) V.

A espessura da camada carburizada foi obtida a partir das metalografias da Figura 1, conforme observado na Tabela 3.

Tabela 3. Espessura carburizada das amostras de estudo.

\begin{tabular}{|c|c|c|}
\hline Amostra & $\begin{array}{c}\text { Estado de } \\
\text { envelhecimento }\end{array}$ & $\begin{array}{c}\text { Camada } \\
\text { carburizada }(\boldsymbol{\mu} \mathbf{m})\end{array}$ \\
\hline A & I & $<50$ \\
\hline B & III & 490 \\
\hline C & V & 750 \\
\hline
\end{tabular}

\subsection{Simulação computacional}

A simulação computacional permitiu determinar a variação da densidade de fluxo magnético na interação entre o yoke (núcleo ferromagnético mais conjunto de ímãs) e o corpo de prova. Foram realizadas mudanças na geometria e nas propriedades magnéticas do modelo levando em consideração a variação da permeabilidade ao longo da espessura do tubo, garantindo assim o melhor acople entre o corpo de prova e o sensor. Uma vez otimizado o modelo, foi determinado o campo magnético em função da variação das propriedades da camada carburizada.

Na Figura 2 apresenta-se o modelo simulado constituído por um núcleo de aço carbono, um conjunto de ímãs (posicionado nas extremidades do núcleo) e o corpo de prova contendo a camada carburizada. As propriedades magnéticas do corpo de prova foram obtidas com o magnetômetro de amostra vibrante (VSM), desta forma, no modelo computacional foram inseridas as curvas $\mathrm{BH}$ correspondentes. Com o objetivo de obter resultados próximos aos valores reais foi utilizada uma malha refinada nos ímãs e na camada carburizada utilizando elementos tetraédricos. 


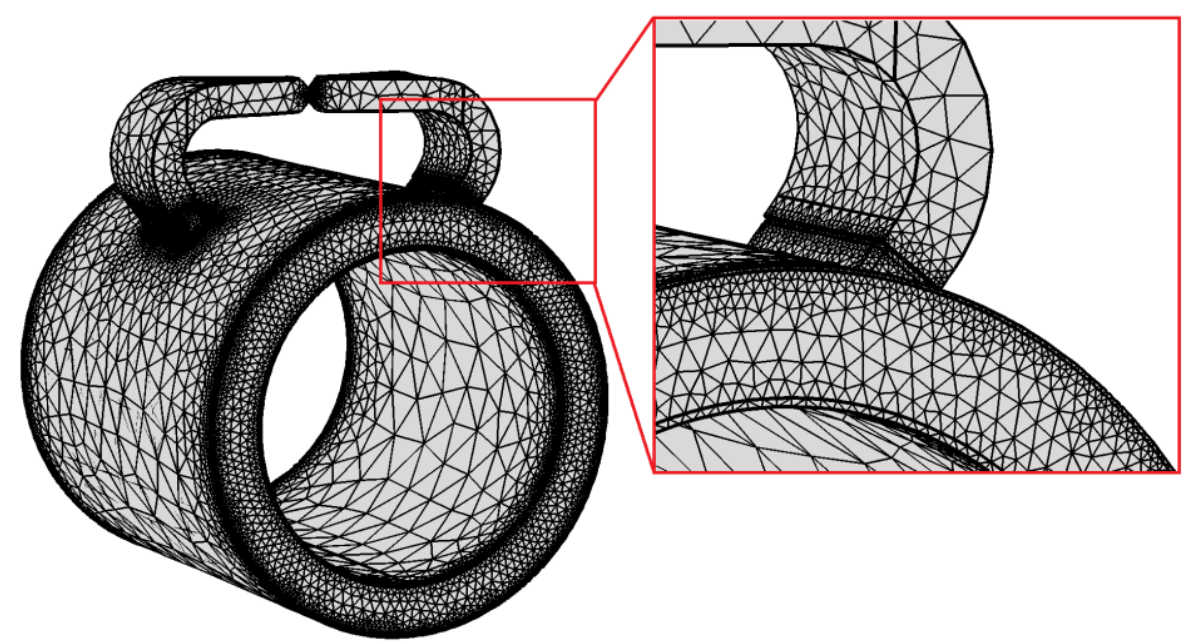

Figura 2. Malha gerada no software de elementos finitos do modelo composto pelo corpo de prova e o sensor de carburização.

A simulação computacional visou estimar a densidade de campo magnético do sensor no ar (valor de referência) e na interação entre o sensor e um corpo de prova com e sem carburização. A amostra utilizada para validar o modelo computacional foi a amostra C. Observa-se na Figura 3 que o campo magnético é conduzido preferencialmente na camada carburizada, fechando o circuito magnético e medindo sua contribuição magnética.

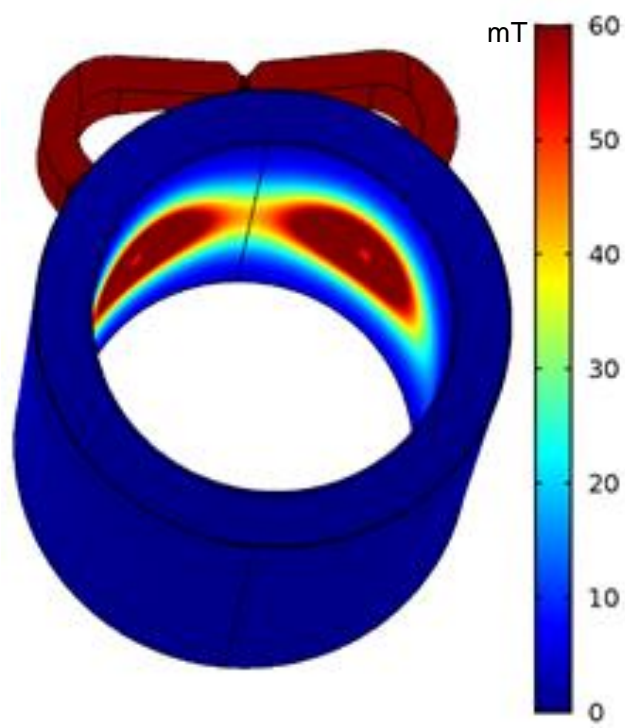

Figura 3. Densidade de campo magnético observado ao longo da espessura do corpo de prova.

Observa-se na Tabela 4 os valores simulados da densidade de campo magnético do corpo de prova $\mathrm{C}$ com e sem carburização.

Tabela 4. Densidade de campo magnético simulada. Valores medidos na parede interna e externa para um corpo de prova com e sem de carburização.

\begin{tabular}{ccc}
\hline $\begin{array}{c}\text { Pontos de } \\
\text { referência }\end{array}$ & $\begin{array}{c}\text { Sem carburização } \\
{[\mathrm{mT}]}\end{array}$ & $\begin{array}{c}\text { Com Carburização } \\
\text { [mT] }\end{array}$ \\
\hline Parede externa & 508,69 & 510,26 \\
Parede interna & 53,56 & 67,69 \\
\hline
\end{tabular}




\subsection{Sensor magnético}

A simulação computacional permitiu construir um sensor de geometria e parâmetros otimizados para a detecção de baixos níveis de carburização em tubos de reforma. De acordo a estes resultados, foi construído um sistema geometricamente simétrico, cujo núcleo ferromagnético consta de uma curvatura suave para evitar perdas e dispersão do campo magnético. Conforme observado na Figura 4, nas suas extremidades é posicionado um arranjo de ímãs. Para detectar a variação do campo magnético é posicionado um sensor de efeito Hall no eixo de simetria do modelo. Com a finalidade de levar o sensor Hall em um ponto de operação linear é posicionado um ímã que serve como bias para diminuir a intensidade do campo magnético na região o sensor.

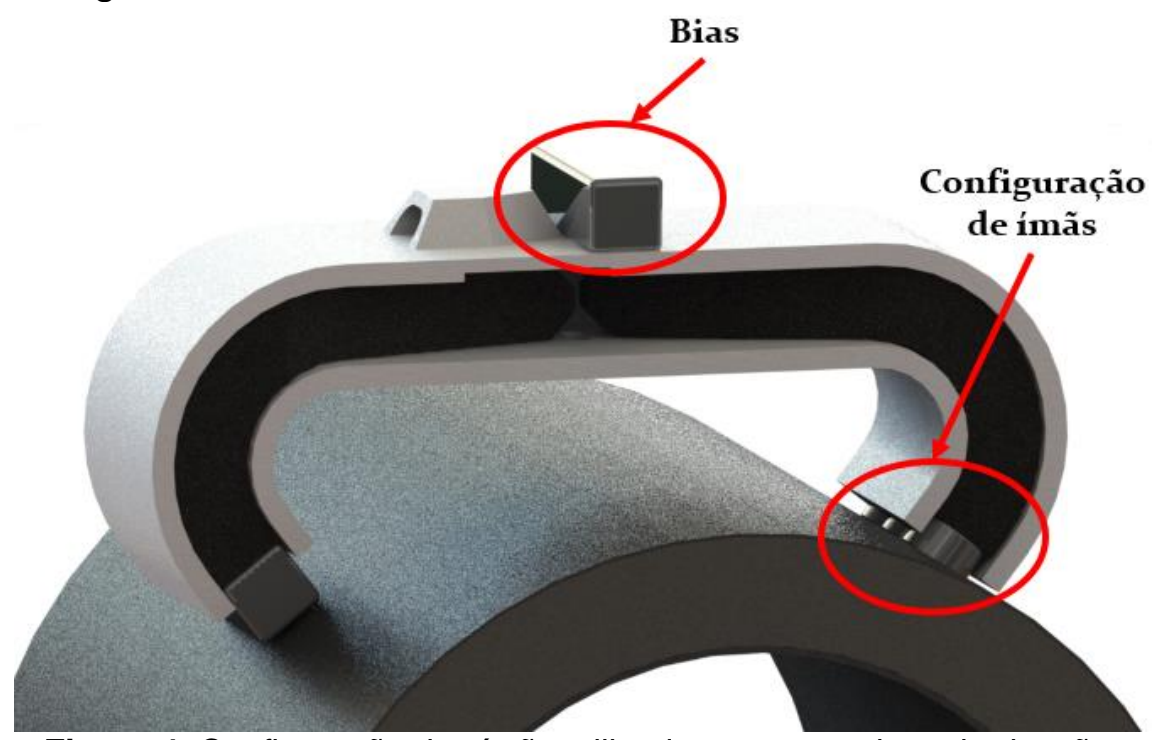

Figura 4. Configuração dos ímãs utilizada no sensor de carburização.

O sensor Hall é alimentado por uma tensão de $5 \mathrm{~V}$ e a leitura da tensão de saída, ou tensão de Hall, é realizada por médio de um voltímetro. Para calibrar o sistema registra-se a tensão de referência do sensor no ar, cujo valor é de aproximadamente $3,5 \mathrm{~V}$. Posteriormente, mede-se a interação entre o sensor magnético e o corpo de prova, o valor de tensão de saída subtraísse do valor de referência. Desta forma, obtém-se a resposta magnética da camada carburizada.

De cada corpo de prova foi retirada uma região carburizada, de aproximadamente $20 \mathrm{~mm}$, com o objetivo de comparar a resposta magnética com e sem carburização. Foi observado que as regiões com carburização apresentaram um sinal de até $60 \%$ mais intenso que a região não carburizada, conforme observado na Figura 5, evidenciando uma resposta ferromagnética na camada carburizada. Avaliando o sinal adquirido nos corpos de prova com camada carburizada foi observado que as amostras A, B e C apresentaram uma tensão de saída de $0,18 \mathrm{mV}, 0,26 \mathrm{mV}$ e 0,52 $\mathrm{mV}$, respectivamente. Estes resultados indicam que na medida que aumenta a espessura da camada carburizada o sinal de saída é mais intenso. 


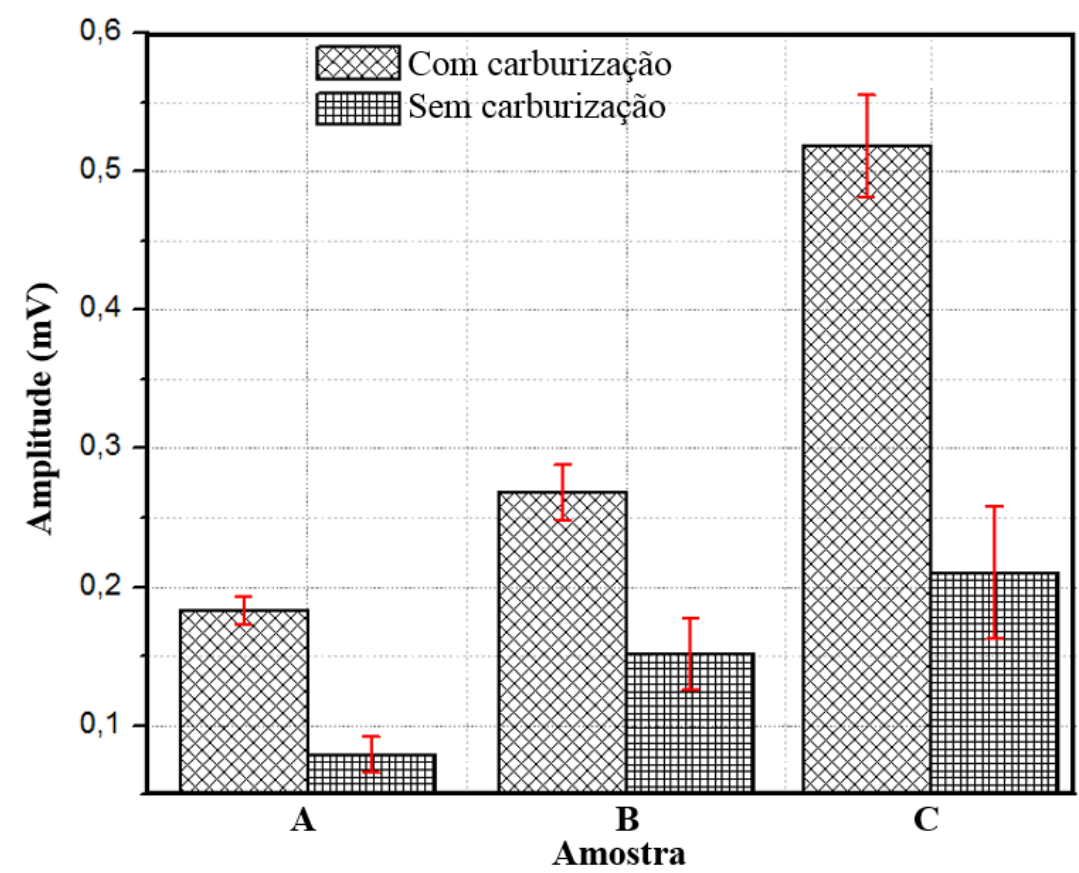

Figura 5. Amplitude da voltagem de saída dos diferentes corpos

\section{CONCLUSÃO}

de prova com e sem região carburizada.

A simulação computacional permitiu otimizar a geometria e as propriedades magnéticas para a construção de um sensor magnético.

O sensor desenvolvido permitiu medir confiavelmente a resposta ferromagnética da camada carburizada.

\section{Agradecimentos}

À CAPES, CNPq e FAPERJ pelo apoio financeiro. À Petrobras pelo fornecimento das amostras. À equipe ensaios não destrutivos do LNDC pelo apoio técnico no desenvolvimento do trabalho.

\section{REFERÊNCIAS}

1 Le May I, Silveira TL da, Vianna CH. Criteria for the Evaluation of Damage and Remaining Life in Reformer Furnace Tubes. Int J Press Vessel Pip. 1996;66(1):233-41.

2 Le May I, Silveira TL da. Reformer Furnaces: Materials, damage mechanisms and assessment. Arab J Sci Eng. 2006;31(2):99-119.

3 Bonaccorsi L, Guglielmino E, Pino R, Servetto C, Sili A. Damage analysis in Fe-Cr-Ni centrifugally cast alloy tubes for reforming furnaces. Eng Fail Anal [Internet]. Elsevier Ltd; 2014;36:65-74. Available from: http://dx.doi.org/10.1016/j.engfailanal.2013.09.020

4 Alvino A, Lega D, Giacobbe F, Mazzocchi V, Rinaldi A. Damage characterization in two reformer heater tubes after nearly 10 years of service at different operative and maintenance conditions. Eng Fail Anal. Elsevier Ltd; 2010;17(7-8):1526-41.

5 Santos YT, Silva GP, Silva IC, Silveira T, Farias CT. Ultrasonic Non-destructive Evaluation of Isothermal Aging in HP Steels. 2014;(Ecndt).

6 Kaya AA. Microstructure of HK40 alloy after high temperature service in oxidizing / carburizing environment II . Carburization and carbide transformations. Mater Charact. 2002;49:23-4. 
7 McLeod AC, Bishop CM, Stevens KJ, Kral MV. Microstructure and Carburization Detection in HP Alloy Pyrolysis Tubes. Metallogr Microstruct Anal. 2015;(60):1020-7.

8 Silva IC, Rebello JMA, Bruno AC, Jacques PJ, Nysten B, Dille J. Structural and magnetic characterization of a carburized cast austenitic steel. Scr Mater. 2008;59(9):1010-3.

9 Stevens K, Tack A, Thomas C, Stewart D. Through-wall carburization detection in ethylene pyrolysis tubes. J Phys D Appl Phys. 2001;34:814-22.

10 ASTM-A297/A297M. Standard Specification for Castings, Iron-Chromium, IronChromium-Nickel, Corrosion Resistant, for General Application. Standard ASTM ASTM International; 2008 p. 1-3. 